\title{
High coverages of hydrogen on a $(10,0)$ carbon nanotube
}

\author{
Charles W. Bauschlicher, Jr. \\ Mail Stop 230-3 \\ NASA Ames Research Center \\ Moffett Field, CA 94035
}

\begin{abstract}
The binding energy of $\mathrm{H}$ to a $(10,0)$ carbon nanotube is calculated at 24,50 , and $100 \%$ coverage. Several different bonding configurations are considered for the $50 \%$ coverage case. Using the ONIOM approach, the average $\mathrm{C}-\mathrm{H}$ bond energy for the most stable $50 \%$ coverage and for the $100 \%$ coverage are 57.3 and $38.6 \mathrm{kcal} / \mathrm{mol}$, respectively. Considering the size of the bond energy of $\mathrm{H}_{2}$, these values suggest that it will be difficult to achieve $100 \%$ atomic $\mathrm{H}$ coverage on a $(10,0)$ nanotube.
\end{abstract}

\section{INTRODUCTION}

We recently ${ }^{1}$ computed the binding energy of hydrogen atoms to the side walls of a $(10,0)$ carbon nanotube using the ONIOM method ${ }^{2-4}$. The first $\mathrm{C}-\mathrm{H}$ bond was $21.6 \mathrm{kcal} / \mathrm{mol}$. The average $\mathrm{C}-\mathrm{H}$ bond strength for the first two hydrogen atoms was $40.6 \mathrm{kcal} / \mathrm{mol}$ and for the first four hydrogens $47.9 \mathrm{kcal} / \mathrm{mol}$. While there is an increase in the bond strength with number of hydrogens, the values were still small compared with the $\mathrm{H}-\mathrm{H}$ bond at the same level of theory $(109.8 \mathrm{kcal} / \mathrm{mol})$. Therefore we suggested that even at high hydrogen coverage the $\mathrm{C}-\mathrm{H}$ bonding would be endothermic or only slightly exothermic. Considering the interest ${ }^{5-7}$ in storing $H$ 
using carbon nanotubes, in this manuscript we report on the results of calculations that model high $\mathrm{H}$ coverages on the same $(10,0)$ carbon nanotube.

\section{MODEL AND METHODS}

The initial coordinates of the $20 \AA$ segment of the $(10,0)$ nanotube were generated using the code of $\mathrm{Han}^{8}$. The dangling bonds at the ends were tied off with hydrogen atoms, yielding a $\mathrm{C}_{200} \mathrm{H}_{20}$ tube. For the $100 \%$ coverage, one additional $\mathrm{H}$ atom is bound to each carbon atom, thus yielding a $\mathrm{C}_{200} \mathrm{H}_{220}$ species, which is shown in Fig. 1. For $24 \%$ and $50 \%$ coverages three random coverage patterns were considered. In each of these, the $\mathrm{H}$ atoms were added in pairs. The first $\mathrm{H}$ atom was added to one of the bare carbon atoms in a random manner. The second $\mathrm{H}$ atom was then added to one of the bare first nearest neighbors in a random manner. If there were no first nearest neighbors, the first $\mathrm{H}$ placement was rejected. This approach was used to break individual C-C $\pi$ bonds, and hence maximize the remaining $\pi$ bonding in the tube.

The observation that $\mathrm{F}$ on the outside $\mathrm{e}^{9}$ and $\mathrm{I}$ on the inside ${ }^{10}$ of carbon nanotubes have distinct patterns, rather than random coverages, leads us to investigate several higher symmetry structures for the $50 \% \mathrm{H}$ coverage; these cases are shown in Figs. 2 to 6. Fig. 2 shows the case where the $\mathrm{H}$ atoms are parallel to the axis of the tube and evenly spaced around the tube. In Fig. 3, the hydrogens are also along the axis of the tube, but the pairs of lines of $\mathrm{H}$ atoms are adjacent. These two structures are denoted "lines" and "pairs of lines", respectively. In Fig. 4, the hydrogens atoms spiral around the tube; this structure is denoted as "spiral". In Figs. 5 and 6 , there are rings of $\mathrm{H}$ atoms; in the first, denoted "rings", the hydrogens are evenly spaced, while in the second case, the rings of hydrogen atoms are in pairs, which is denoted as "pairs of rings".

The AM1 and two level ONIOM approach ${ }^{2-4}$ are used. The ONIOM approach 
combines the Universal Force Field ${ }^{11}$ (UFF), for the low-level treatment, with the hybrid $^{12}$ B3LYP ${ }^{13}$ functional, for the high-level description. The $4-31 \mathrm{G}$ basis set ${ }^{14}$ is used in conjunction with the B3LYP calculations. The AM1 calculations are performed using a modified version of Gaussian 94, where damping is used to obtain convergence. ONIOM calculations are performed using Gaussian $98^{15}$.

The geometries are fully optimized at the AM1 and ONIOM levels of theory. In the ONIOM calculations, twenty-four carbon atoms, at the center of the nanotube, are used for the high-level treatment. The boundary hydrogen atoms and the chemisorbed hydrogen atoms are also in the high level treatment. The atoms included in the high level treatment are the same as used in our previous treatment ${ }^{1}$ of the low coverage case. We should note that more recent work has shown ${ }^{16}$ that capping the carbon nanotubes instead of terminating the dangling bonds with hydrogen atoms appears to dramatically speed up the geometry optimization process.

As noted in our previous study, we encounted problems with local minima for the UFF description of the carbon nanotube, and therefore we use the B3LYP energies instead of the ONIOM energies, since the B3LYP energies were insensitive to the UFF solution. That is, we only use the molecular mechanics approach to constrain the shape of the high-level fragment.

\section{RESULTS AND DISCUSSION}

We first consider the results obtained at the AM1 level of theory-see Table I. The average $\mathrm{H}$ binding energy for three $24 \%$ random coverages span a range of about $5 \mathrm{kcal} / \mathrm{mol}$. Since there are $48 \mathrm{C}-\mathrm{H}$ bonds, the stability of the tubes vary by $243 \mathrm{kcal} / \mathrm{mol}$. The average $\mathrm{H}$ binding energy for the three random $50 \%$ coverages spans a somewhat smaller range, and the values are similar to those obtained for the $24 \%$ coverage. We can conclude that the AM1 binding energies for the 24 and $50 \%$ coverages are similar, but it would require many more runs to more accurately 
determine the average bond energies for these two cases and how it changes between them. However, as discussed below, we can get some insight into the bonding using only these few cases.

In light of patterns observed for $F$ and I with nanotubes, rather than consider more random coverages, we studied five higher symmetry patterns, which are shown in Figs. 2-6 and their average $H$ binding energies are summarized in Table I. Three of the higher symmetry structures are more stable than the random coverages. Considering that there are $100 \mathrm{C}-\mathrm{H}$ bonds, the change in the average $\mathrm{H}$ binding energy means that the three higher symmetry structures are several $100 \mathrm{kcal} / \mathrm{mol}$ more stable than the random structures.

The lower stability of the random structures arises for two reasons: 1) there are areas without $\mathrm{H}$ atoms that contain an odd number of carbons, which dramatically degrades the $\pi$ bonding, and 2) to form a good C-H bond, the carbon must $\mathrm{sp}^{3}$ hybridize, which results in the carbon bulging out of the tube. For the random tubes, there are areas of very high $H$ coverage, and clearly the tube cannot deform such that every atom can form a good $\mathrm{sp}^{3}$ hybrid bond with $\mathrm{H}$.

For the higher symmetry cases, all regions devoid of $\mathrm{H}$ atoms contain an even number of carbon atoms, so the $\pi$ bonding is not degraded for this reason. For the pairs of lines tube, two rows of carbons bulge out of the tube allowing the formation of good C-H bonds. This deformation of the carbon tube leaves the bare rows of carbon atoms nearly planar; thus this configuration has both good $\mathrm{C}-\mathrm{H}$ bonds and good C-C $\pi$ bonds. In the spiral, the deformation leaves a spiral of good $\mathrm{C}-\mathrm{H}$ bonds and a spiral of $\mathrm{C}-\mathrm{C} \pi$ bonds. In the lines configuration, the geometry appears very favorable for good bonding, but the C-C $\pi$ bonds are isolated, which removes the $\pi$ conjugation, and hence this is less favorable than the pairs of lines or spiral. In the two ring forms, the curvature of tube weakens the $\mathrm{C}-\mathrm{C} \pi$ bonding, thus these forms are unfavorable, even though they distort to form good $\mathrm{C}-\mathrm{H}$ bonds. 
We studied the two most stable $50 \%$ coverage structures, namely the spiral and pairs of lines, using the ONIOM approach. We computed the average $\mathrm{H}$ binding energy of the 12 hydrogens included in the high-level treatment-see Table I. While we do not compute the $\mathrm{H}$ binding energy of the hydrogens in the low-level treatment, their effect on the geometry of the tube is included. The pair of lines ONIOM value is very similar to the AM1 result, while for the spiral, the ONIOM value is somewhat smaller than the AM1 result. On the basis of our low coverage work, part of the difference between the AM1 and ONIOM values can probably be attributed to the fact that many of the $\mathrm{H}$ atoms in the high level treatment are near the boundary between the high and low-levels of theory. However, it seems unlikely that this can account for the entire difference, and some, if not most, of the difference must be attributed to limitations in the methods used, with the AM1 treatment having a larger uncertainty than the ONIOM approach.

Using the ONIOM approach, we computed the average $\mathrm{H}$ binding energy for $100 \%$ coverage, and we find that this value is significantly smaller than the $50 \%$ coverages. For the $100 \%$ coverage, the carbon atoms still form a good tube structure since any deformation that improves one $\mathrm{C}-\mathrm{H}$ bond will weaken another. This means that for the $100 \%$ coverage the carbons cannot change their hybridization to enhance the $\mathrm{C}-\mathrm{H}$ bond, which results in a much weaker $\mathrm{C}-\mathrm{H}$ bond than the $50 \%$ coverage, where half of the $\mathrm{C}$ atoms can bulge out of the tube to maximize the $\mathrm{C}-\mathrm{H}$ bonding.

Despite any limitations in the methods used, it is clear that the formation of $100 \%$ coverage will be a very endothermic process (remember that the $\mathrm{H}-\mathrm{H}$ bond energy is $109.8 \mathrm{kcal} / \mathrm{mol}$ at this level of theory). The computed binding energies for the $50 \%$ coverage cases are sufficiently close to one half the $\mathrm{H}-\mathrm{H}$ bond energy that it might be possible to achieve this level of coverage in an exothermic process starting with $\mathrm{H}_{2}$ and nanotubes. The formation of significantly higher than $50 \%$ coverages in an exothermic process seems unlikely since higher coverages would require a deformation 
of the carbons in the tube that would weaking some of the existing $\mathrm{C}-\mathrm{H}$ bond and hence result in a smaller average $\mathrm{H}$ binding energy.

It should be noted that all of our discussion is for the case of $\mathrm{H}$ atoms on the outside of the tube. It might be possible to achieve high coverage by bonding some $\mathrm{H}$ atoms on the inside of the tube and some on the outside. For example, one half the $\mathrm{C}$ atoms bulge out and bond to $\mathrm{H}$ atoms and the other half of the $\mathrm{C}$ atoms bulge in and bond to $\mathrm{H}$ atoms on the inside of the tube. However, this approach faces the problem of opening the tubes and getting $\mathrm{H}$ atom inside.

\section{CONCLUSIONS}

We use the AM1 level of theory to obtain some insight into the bonding of $\mathrm{H}$ atoms to a $(10,0)$ carbon nanotube. The carbon atoms that bond to the $\mathrm{H}$ atoms bulge out of the tube to improve the $\mathrm{sp}^{3}$ hybridization, which enhances the $\mathrm{C}-\mathrm{H}$ bond. For $50 \%$ coverage we have found two structures that have half of the $\mathrm{C}$ atoms bulged out of the tube to form good C-H bonds, while the remaining carbons atoms can still have good $\pi$ bonding. Random coverages are found to be much less favorable because not all atoms have a favorable orientation for either $\mathrm{C}-\mathrm{H}$ or $\mathrm{C}-\mathrm{C} \pi$ bonding. Random coverage also tends to lead to bare regions with an odd number of carbon atoms which is not ideal for $\pi$ bonding. Using the ONIOM approach, the average C-H bond energy was computed for the two most favorable $50 \%$ coverage cases as well as for $100 \%$ coverage. It is possible that the formation of $50 \%$ coverage is thermodynamically favorable, but it is concluded that the formation of $100 \%$ coverage will be very endothermic. 


\section{REFERENCES}

${ }^{1}$ C. W. Bauschlicher, Chem. Phys. Lett. 322 (2000) 237.

${ }^{2}$ M. Svensson, S. Humbel, R. D. J. Froese, T. Matsubara, S. Sieber, K. Morokuma, J. Phys. Chem. 100 (1996) 19357.

${ }^{3}$ S. Humbel, S. Sieber, K. Morokuma, J. Chem. Phys. 105 (1996) 1959.

${ }^{4}$ F. Maseras, K. Morokuma, J. Comp. Chem. 16 (1995) 1170.

${ }^{5}$ P. Chen, X. Wu, J. Lin, K. L. Tan, Science, 285 (1999) 91.

${ }^{6}$ Y. Ye, C.C. Ahn, C. Witham, B. Fultz, J. Liu, A.G. Rinzler, D. Colbert, K.A. Smith, R.E. Smalley, Appl. Phys. Lett. 74 (1999) 2307.

${ }^{7}$ V. V. Simonyan, P. Diep, J. K. Johnson, J. Chem. Phys. 111 (1999) 9778.

${ }^{8} \mathrm{~J}$. Han, personal communication.

${ }^{9}$ Kelly, K. F.; Chiang, I. W.; Mickelson, E. T.; Hauge, R. H.; Margrave, J. L.; Wang, X.; Scuseria, G. E.; Radloff, C.; Halas, N. J. Chem. Phys. Lett. 1999, 313, 445.

${ }^{10}$ X. Fan, E. C. Dickey, P. C. Eklund, K. A. Williams, L. Grigorian, R. Buczko, S. T. Pantelides, and S. J. Pennycook, Phys. Rev. Lett. 84 (2000) 4621.

${ }^{11}$ Rappe, A. K.; Casewit, C. J.; Colwell, K. S.; Goddard, W. A.; Skiff, W. M. J. Am. Chem. Soc. 1992, 114, 10024.

${ }^{12}$ Becke, A. D. J. Chem. Phys. 1993, 98, 5648.

${ }^{13}$ Stephens, P. J.; Devlin, F. J.; Chabalowski, C. F.; Frisch, M. J. J. Phys. Chem. $1994,98,11623$.

${ }^{14}$ Frisch, M. J.; Pople, J. A.; Binkley, J. S., J. Chem. Phys. 1984, 80, 3265 and references therein. 
${ }^{15}$ Gaussian 98, Revision A.7, M. J. Frisch et al., Gaussian, Inc., Pittsburgh PA, 1998.

${ }^{16} \mathrm{C}$. W. Bauschlicher, unpublished. 


\section{TABLES}

TABLE I. Summary of hydrogen binding energies, in $\mathrm{kcal} / \mathrm{mol}$.

\begin{tabular}{|c|c|}
\hline System & Average $\mathrm{H}$ binding energy \\
\hline \multicolumn{2}{|l|}{ AM1 } \\
\hline $24 \%$ random case 1 & 53.80 \\
\hline $24 \%$ random case 2 & 52.08 \\
\hline $24 \%$ random case 3 & 48.74 \\
\hline $50 \%$ random case 1 & 51.11 \\
\hline $50 \%$ random case 2 & 50.67 \\
\hline $50 \%$ random case 3 & 48.91 \\
\hline $50 \%$ pairs of lines & 58.29 \\
\hline $50 \%$ spiral & 57.62 \\
\hline $50 \%$ lines & 56.11 \\
\hline $50 \%$ rings & 51.51 \\
\hline $50 \%$ pairs of rings & 48.54 \\
\hline \multicolumn{2}{|l|}{ ONIOM } \\
\hline $1 \mathrm{H}^{\mathrm{a}}$ & 21.6 \\
\hline $2 \mathrm{H}^{\prime} \mathrm{s}^{a}$ & 40.6 \\
\hline $4 \mathrm{H}^{\prime} \mathrm{s}^{\mathrm{a}}$ & 47.9 \\
\hline $50 \%$ pairs of lines & 57.29 \\
\hline $50 \%$ spiral & 52.64 \\
\hline $100 \%$ & 38.65 \\
\hline
\end{tabular}

a Taken from reference 1. 


\section{FIGURES}

FIG. 1. The $\mathrm{C}_{200} \mathrm{H}_{220}$ tube used to model $100 \%$ hydrogen coverage on a $(10,0)$ carbon nanotube.

FIG. 2. The $\mathrm{C}_{200} \mathrm{H}_{120}$ tube used to model the "lines" form of the $50 \%$ hydrogen coverage on a $(10,0)$ carbon nanotube.

FIG. 3. The $\mathrm{C}_{200} \mathrm{H}_{120}$ tube used to model the "pairs of lines" form of the $50 \%$ hydrogen coverage on a $(10,0)$ carbon nanotube.

FIG. 4. The $\mathrm{C}_{200} \mathrm{H}_{120}$ tube used to model the "spiral" form of the $50 \%$ hydrogen coverage on a $(10,0)$ carbon nanotube.

FIG. 5. The $\mathrm{C}_{200} \mathrm{H}_{120}$ tube used to model the "rings" form of the $50 \%$ hydrogen coverage on a $(10,0)$ carbon nanotube.

FIG. 6. The $\mathrm{C}_{200} \mathrm{H}_{120}$ tube used to model the "pairs of rings" form of the $50 \%$ hydrogen coverage on a $(10,0)$ carbon nanotube. 


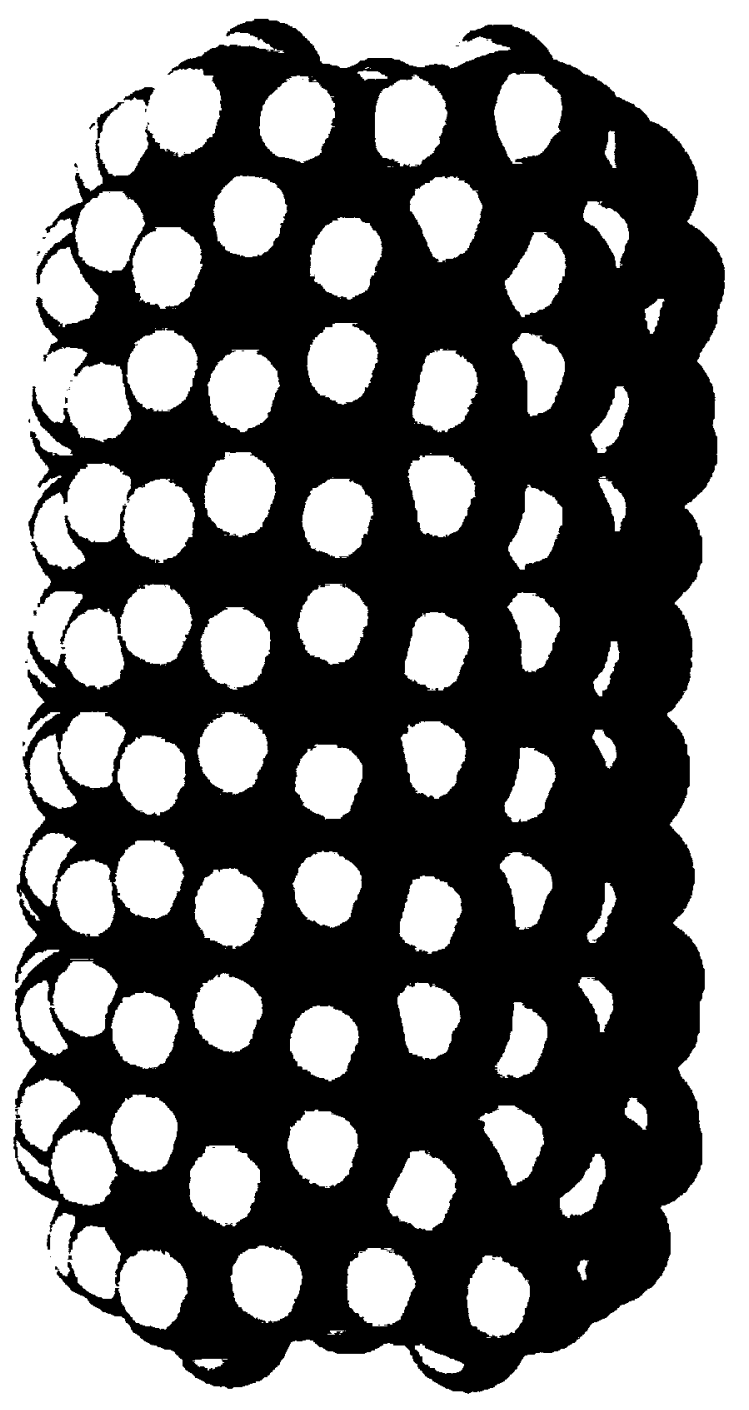

f

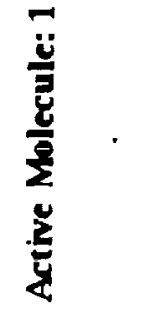




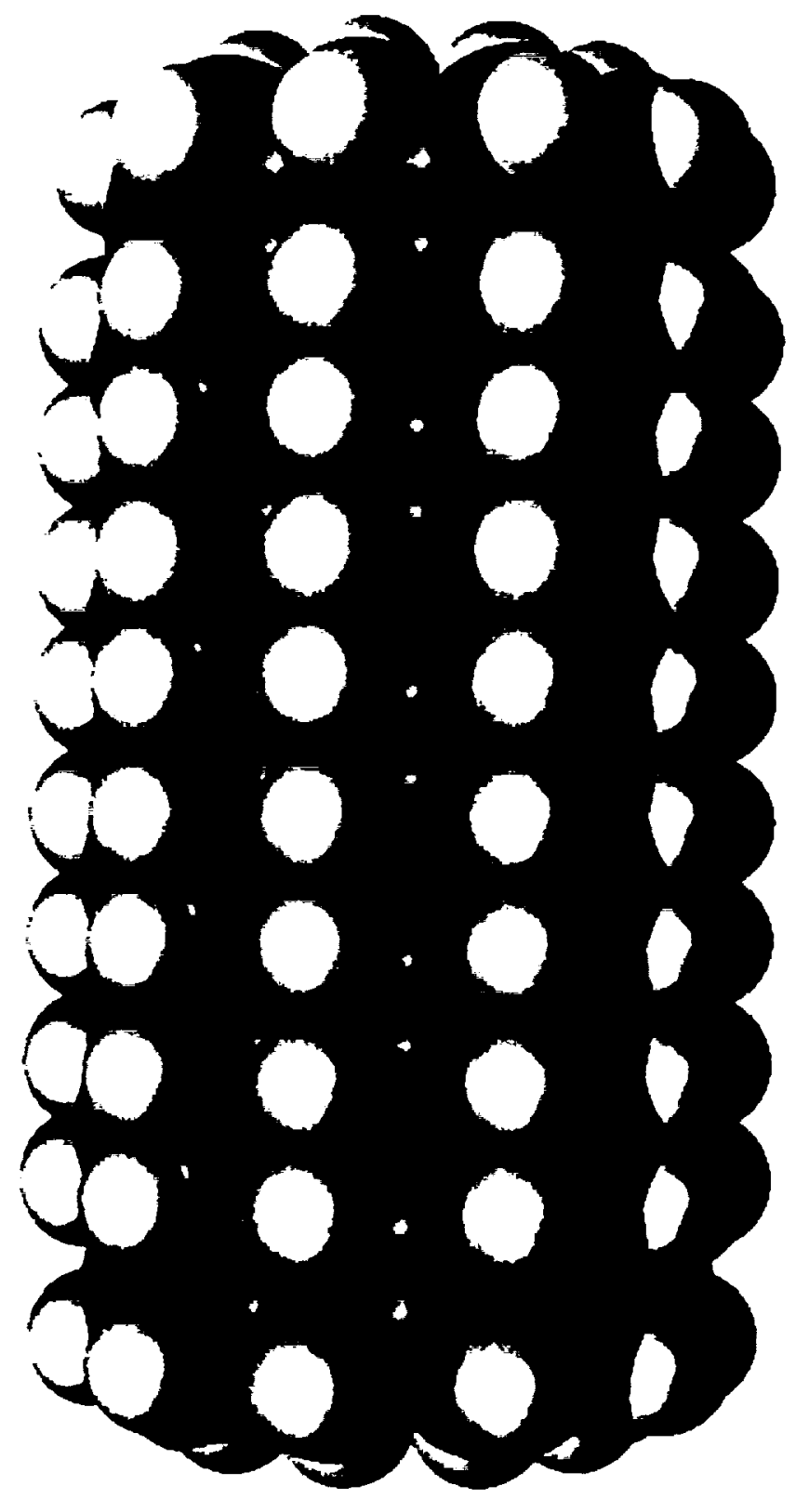

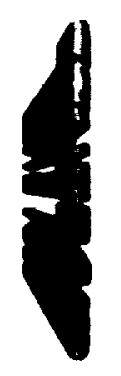

$\frac{\vec{z}}{3}$ 


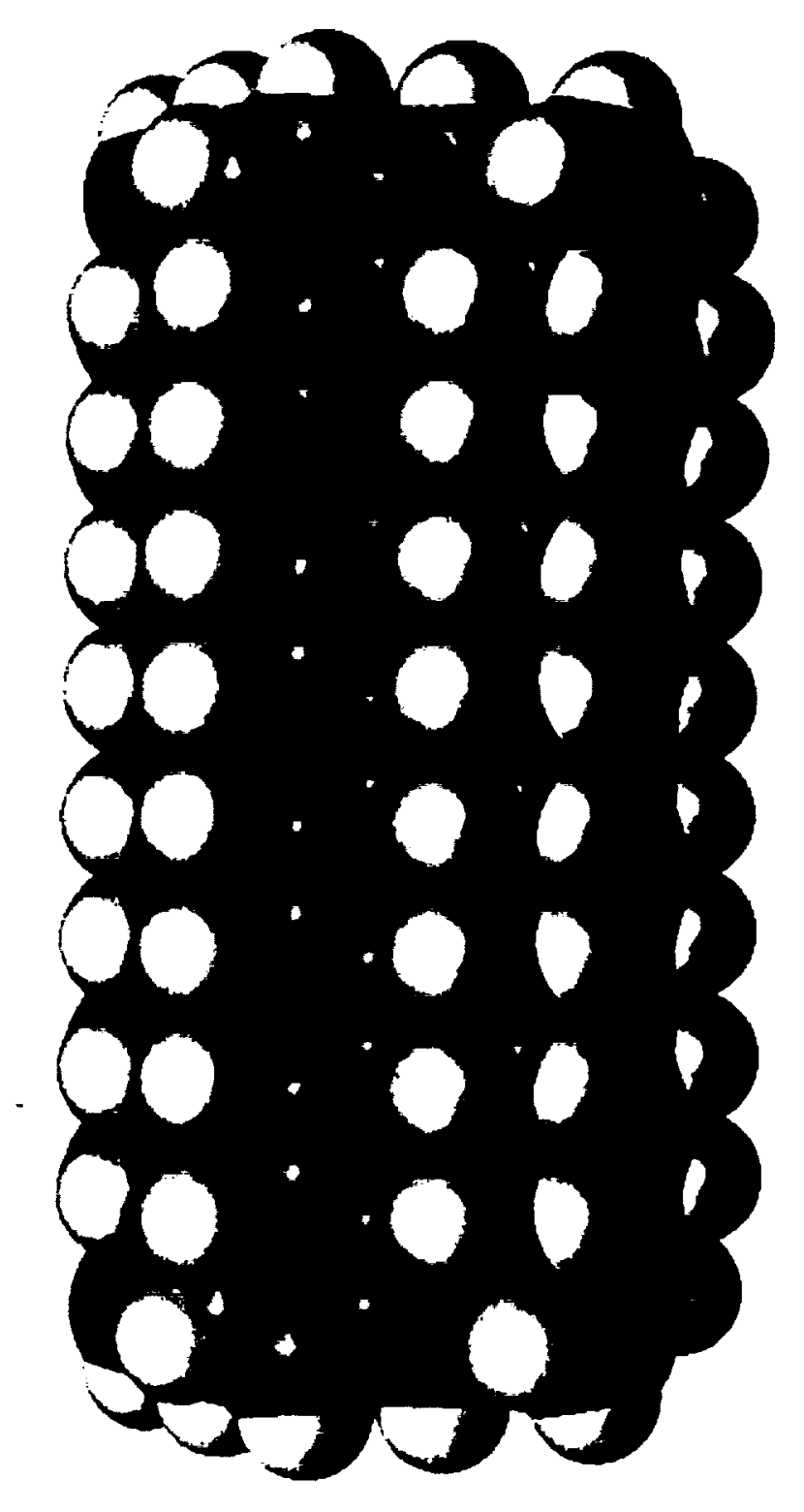

8

$\frac{7}{\frac{8}{3}}$ 


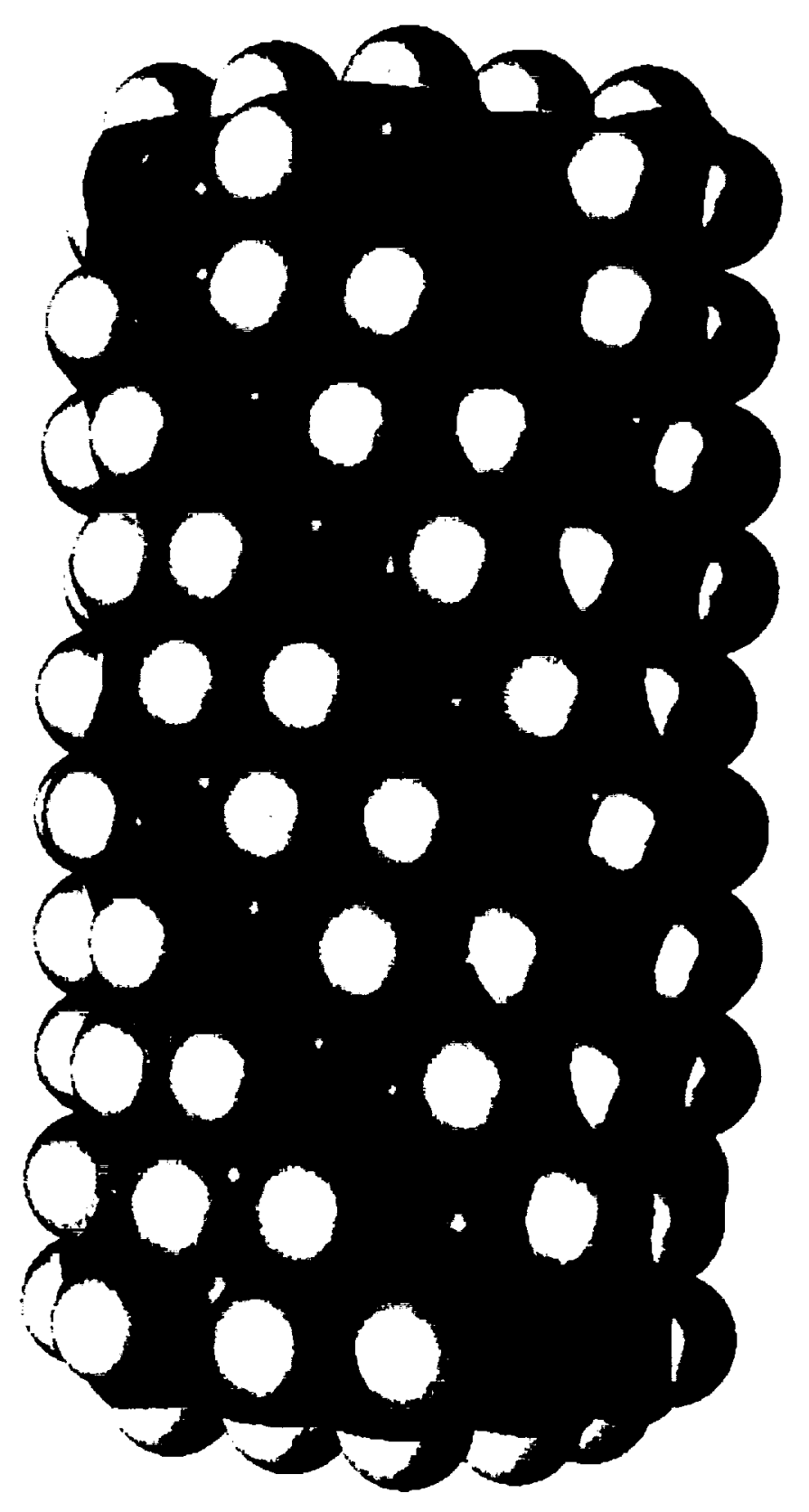

8

$\frac{7}{3}$ 

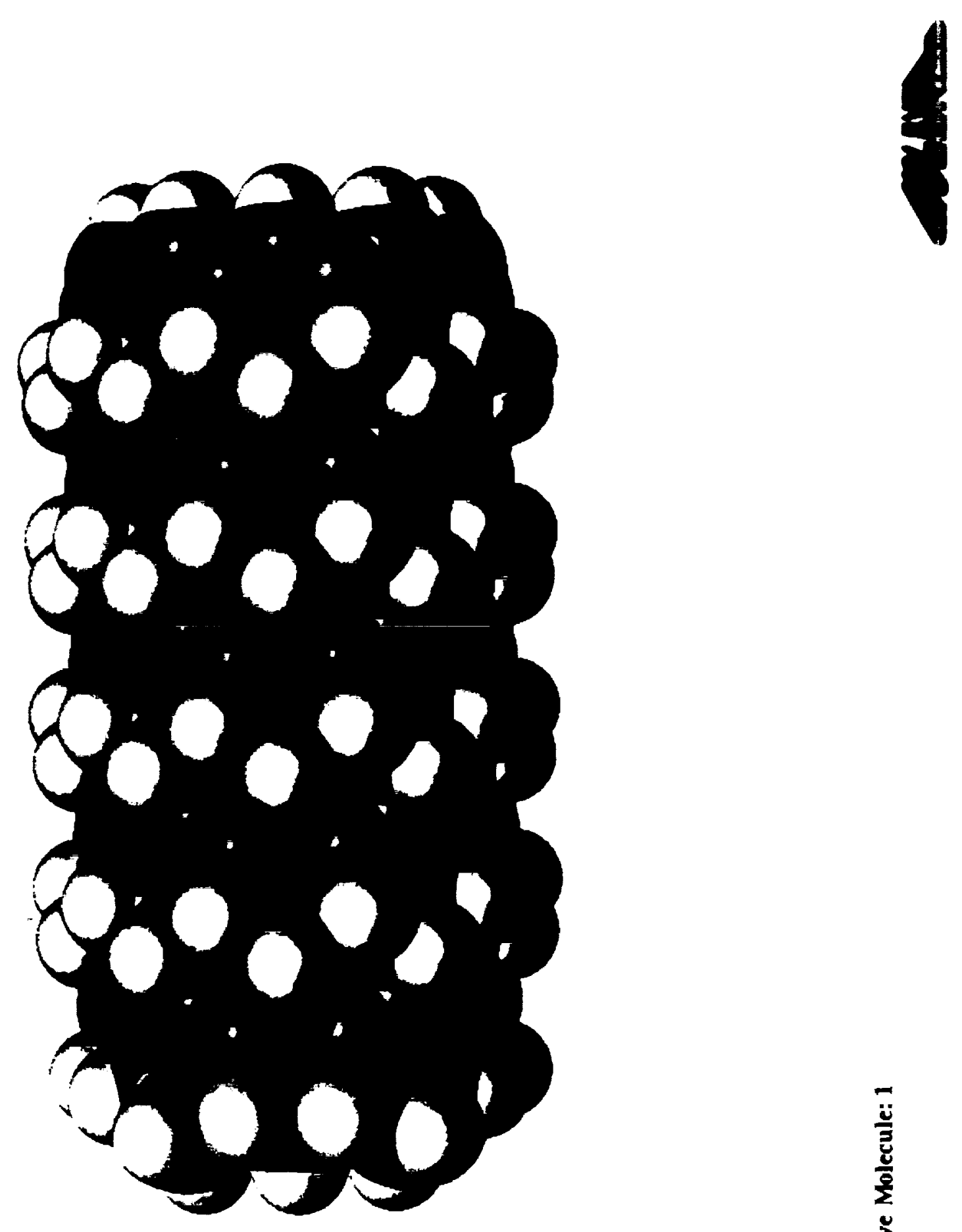

$\frac{\bar{z}}{\frac{8}{3}}$ 


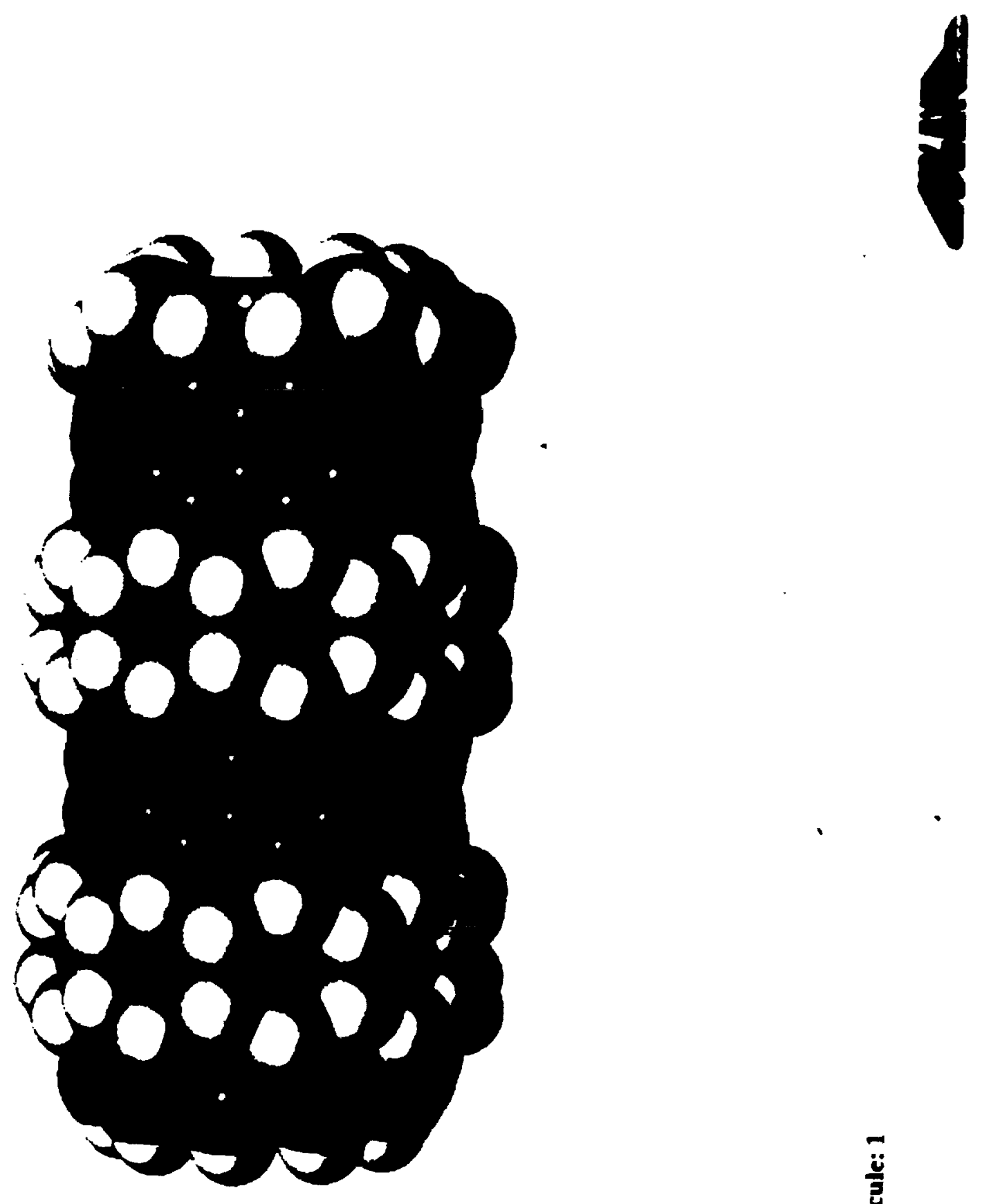

童. 